\title{
Perspectivas en el estudio de las Pteridofitas
}

\author{
RAMÓN RIBA Y BLANCA PÉREZ GARCÍA ${ }^{1}$
}

'Departamento de biología, C.B.S. Universidad Autónoma Metropolitana-Iztapalapa. Apdo. Postal 55-535 09340, México D.F.

\begin{abstract}
Resumen. Las pteridofitas han sido estudiadas en sus inicios principalmente con base en la morfología del esporofito, en la que descansan las principales clasificaciones. Como resultado de los avances en otras disciplinas, y las mejoras tecnológicas en sistemas de observación y técnicas de laboratorio, en nuestros días hay una clara y evidente interrelación disciplinaria que ha llevado a revisar viejos conceptos con la consecuente modificación de ideas y teorías de la biología de las pteridofitas. En este artículo se pretende mostrar el desarrollo de la taxonomía, desde sus inicios hasta los diversos enfoques con que son abordados en la actualidad los problemas que plantea. Para ello se hará mención de las principales líneas de trabajo que se siguen, así como las contribuciones más importantes en cada campo de investigación.
\end{abstract}

Abstract. In the beginnings, the study of the pteridophytes was supported mainly in the morphology of the sporophyte. As a result of the development and the advances in other disciplines and the technological improvements in observation systems as well as field and laboratory techniques, a clear and evident disciplinary interrelationship has arisen and a revision of old concepts has been necessary with the consequent modification of ideas and theories about the biology of the pteridophytes. We intend to show the development of the taxonomy of the pteridophytes from the early days to the different modern approaches used today to understand the present problems through the principal fields of research and the most significative contributions.

\section{INTRODUCCIÓN}

Las plantas han sido objeto de la curiosidad de los seres humanos prácticamente desde que éstos aparecieron sobre la Tierra, cuando por necesidad obtenían sus alimentos de la vida silvestre y, por lo consiguiente, eran taxónomos prácticos. «...Por medio de la experiencia, estos antiguos antepasados nuestros aprendieron a distinguir a las plantas comestibles de las que no lo eran. Y (como alguien dijo), aquellos que no pudieron aprender esos elementos de la Botánica, tampoco pudieron ser nuestros antepasados» (Radford et al., 1974).

Desde esos lejanos tiempos hasta nuestros días han transcurrido miles de años, y se ha pasado del «conocimiento» por vez primera al «reconocimiento» por comparación, y finalmente al «estudio», que no tiene solamente fines utilitarios.

Los primeros estudios sobre las pteridofitas que pueden ser llamados formales, tuvieron un enfoque taxonómico, aunque por el desconocimiento de su ciclo de vida y de otras peculiaridades como su sistema vascular, adolecieron de muchas inconsistencias. Poco a poco, esos estudios se fueron afinando gracias al desarrollo de otras disciplinas y a las interrelạciones que se establecían, hasta llegar a sistemas de clasificación más coherentes. Por esta razón mencionamos en seguida algunos de los hechos más significativos en el estudio de estas plantas, sin tratar de hacer una revisión histórica completa como la realizada ya por Pichi Sermolli (en: Jermy et al., 1973). De igual manera, al abordar las distintas disciplinas a través de las cuales han sido estudiadas las pteridofitas, se ha hecho una selección de los trabajos más relevantes de cada una de ellas.

\section{ANTECEDENTES TAXONÓMICOS HISTÓRICOS}

Algunos testimonios escritos que pueden ser considerados como académicos sobre las pteridofitas, datan de la segunda mitad del siglo XVI, cuando en su obra De Plantis Libris XVI (1583) Cesalpino incluyó en un solo grupo a helechos, equisetos, licopodios (Selaginella), musgos, algas y líquenes en la primera clasificación basada en características vegetativas y reproductivas.

Un siglo después, y pasando por alto clasificaciones muy artificiales de pteridofitas, hubo avances importantes por la utilización de medios ópticos para detallar rasgos de indusios, esporangios, esporas y tipos de dehiscencia (Grew, 1672 y 1682; Malpighi, 1675; Ray, 1686-1704). Morison (1699) fue el primero en describir la germinación de las esporas de los helechos.

Cien años más tarde, Linnaeus (1753) incluyó a las pteridofitas en la clase XXIV (Cryptogamia) de su obra Species Plantarum. Equisetum e Isoëtes están entre los Filices y Lycopodium en Musci.

En 1763, Adanson presentó una clasificación avanzada de plantas con flores, que aunque representaba un retroceso respecto a la de pteridofitas, destacaba la importancia de la ausencia o presencia de indusio como un carácter útil en la clasificación de géneros.

En las primeras décadas del siglo XIX, se comenzó a afinar el concepto de familia en el mismo sentido taxonómico actual. Destacan Brown (Marsileaceae, Osmundaceae; 1810), Berchtold y Presl (Marattiaceae, Osmundaceae, Polypodiaceae; 1820), Agardh (Danaeaceae, Ophioglossaceae; 1822), Presl (Gleicheniaceae; 1825) y Kaulfuss (Cyatheaceae, Schizaeaceae; 1827). 
En 1836, Presl presentó un nuevo sistema de clasificación, en el que utilizó características de soros y esporangios; incluía rasgos vegetativos, morfológicos y anatómicos, venación de las hojas y haces vasculares del pecíolo. A pesar de los avances mostrados en esta clasificación, su valor no fue apreciado sino hasta casi cien años más tarde por la influencia que tuvo en ese tiempo Hooker (1838-1842), quien era partidario de grandes géneros. Otros autores que, a la mitad del siglo XIX, contribuyeron signifi-cativamente a la taxonomía de las pteridofitas fueron Fée (1852), quien siguió los criterios de Presl en la utilización de características anatómicas y morfológicas para la taxonomía de los helechos; Mettenius $(1856,1858,1859)$, que aunque conservador en la delimitación de géneros, produjo una clasificación de familias de helechos en la que las afinidades entre ellas se expresaban de manera muy adelantada para su época, y finalmente Smith (1842-1843 ) y Moore (1857-1862), quienes contribuyeron a la taxonomía a nivel de género.

La última parte del siglo XIX se caracterizó por los intentos de entender a la taxonomía con base en los conceptos de la evolución. Para fines de ese siglo, los hallazgos derivados del estudio del ciclo de vida de las plantas, habían llevado al reconocimiento de dos fases diferentes desde el punto de vista de la morfología y de la citología. Entonces, en 1894, Strasburger reconoció los dos rasgos clave en ese ciclo de vida, la fecundación y la meiosis.

A principios del siglo $\mathrm{XX}, \mathrm{y}$ como resultado del desarrollo de los conceptos filogenéticos, se revivió el interés en la taxonomía de las pteridofitas. Christensen publicó el Index Filicum (1905-1906), seguido de tres suplementos $(1913,1917,1934)$, en los que hace una recopilación de los géneros y especies conocidos hasta entonces. Y aunque no es innovador en lo que se refiere a la taxonomía de los grupos superiores de helechos, pues sigue el sistema del Pflanzenfamilien (Engler y Prantl, 1898-1902), es muy útil en la taxonomía de los géneros y especies.

En 1908, Bower publicó The Origin of a Land Flora, obra en la que virtió sus profundos conocimientos acerca de la morfología y anatomía de las dos fases del ciclo de vida de las plantas arquegoniadas. Su obra culmina cuando, entre 1923 y 1928, publicó The Ferns. Aquí se condensó la información obtenida en más de treinta años de investigaciones de asuntos morfológicos, morfogenéticos y anatómicos, y ha sido una invaluable fuente de información para las generaciones posteriores de pteridólogos, e influyó fuertemente en la pteridología durante la primera mitad del siglo XX.

Este resumen de los principales hechos en los estudios taxonómicos sobre pteridofitas, pone de manifiesto que en sus principios la taxonomía se basó de manera natural en la organización de grupos que, en muchos casos, resultaron artificiales, dado que las plantas eran agrupadas por similitudes aunque éstas no reflejaran afinidades reales. De cualquier manera, se establecieron las bases para el desarrollo de otras líneas de investigación que, de forma obligada, serían útiles para las interpretaciones taxonómicas de las pteridofitas.
La diversidad de enfoques mediante los cuales las pteridofitas son estudiadas actualmente, será analizada en el siguiente apartado. Sin pretender hacer una revisión exhaustiva, presentamos las líneas que consideramos sobresalientes, con la mención de los principales grupos de trabajo.

\section{LÍNEAS DE INVESTIGACIÓN}

Las pteridofitas han sido estudiadas desde muy diversos enfoques disciplinarios, los que en su inicio se desarrollaron de manera independiente. A medida que las distintas disciplinas se fueron interrelacionando, se hizo necesario revisar de manera conjunta el estado en que se encontraba el estudio de la biología de estas plantas, por lo que en 1972 se llevó a cabo en Londres un simposio sobre filogenia y clasificación de Filicopsida. Las presentaciones fueron publicadas in extenso en 1973 como Suplemento 1 del Botanical Journal of the Linnean Society (Jermy et al., Edrs.). En esta publicación se reflejaron ideas de las tendencias que había en esas fechas acerca de la evolución y la sistemática de Filicopsida. Se puso en evidencia la importancia de considerar aspectos geológicos, ontogenéticos y ecológicos como partes importantes de la sistemática.

Seis años después, en 1979, Dyer editó The Experimental Biology of Ferns. En las numerosas contribuciones se presentaron investigaciones sobre aspectos reproductivos como apogamia y aposporia, dispersión y germinación de esporas, establecimiento de gametofitos, diferenciación por medio de controles químicos, ecología, etc. En este volumen no estaban representados de manera adecuada, ni estudios de campo o poco experimentales, $\mathrm{ni}$ aspectos relacionados con las plantas afines a los helechos. Esta deficiencia se subsanó pocos años más tarde.

En 1983, se llevó a cabo en Edimburgo un simposio sobre Biología de Pteridofitas. En él se abordaron problemas relacionados con la biología de los helechos y plantas afines a ellos, y las contribuciones presentadas se publicaron en el libro Biology of Pteridophytes, editado por Dyer y Page (1985).

Hasta esas fechas, la fase dominante en el ciclo de vida de los helechos y plantas afines, el esporofito, había recibido atención preponderante en libros y monografías de carácter morfológico, filogenético y evolutivo, lo que se ha visto reflejado fundamentalmente en las múltiples clasificaciones existentes. Los gametofitos, a pesar de que ya Morison (1699) había escrito sobre ellos, fueron objeto de estudios esporádicos durante más de 200 años, y no fue sino hasta las contribuciones de Stokey (1918) en la primera mitad del siglo XX cuando comenzó a verse la posibilidad de usarlos como instrumentos para consideraciones filogenéticas y taxonómicas. La publicación de Miller, Fern Gametophytes as Experimental Material (1968), es una recopilación de los estudios hechos sobre factores bióticos y abióticos que influyen en la germinación, viabilidad, citología y polaridad de las esporas de helechos, cultivo de tejidos y diferenciación de gametangios y otros aspectos reproductivos. Se pone de manifiesto la importancia de este material biológico 
como modelo experimental, para entender diversos procesos en otros grupos de plantas.

En 1989, Raghavan publicó Developmental Biology of fern gametophytes. En este libro se describe cómo la espora origina el plan estructural básico del gametofito, cómo crece y se reproduce y finalmente cómo se inicia la fase esporofítica. En los primeros capítulos se discute el potencial bioquímico de la espora que se refleja en células destinadas a desarrollar diferentes funciones; los siguientes capítulos revisan los procesos del crecimiento del gametofito hasta alcanzar el estado laminar, después se analizan los procesos relacionados con la inducción de la sexualidad y de la diversidad genética, y finalmente se examinan los procesos de apogamia y de aposporia en los helechos y sus implicaciones en la citología de estas plantas.

\section{TAXONOMÍA}

Los esfuerzos para encontrar un sistema de clasificación que reflejara las relaciones filogenéticas entre los grandes grupos de pteridofitas, condujeron a la presentación de clasificaciones en las que eran consideradas como integrantes de una sola división, separando los grupos como clases de la misma, hasta aquellas que consideran a estos grupos como divisiones independientes. De manera simultánea, se han hecho innumerables revisiones taxonómicas a nivel de familia sobre todo en los helechos polipodiáceos, con distintos arreglos y distribución de géneros en las mismas. De igual forma, han sido publicadas numerosas revisiones de géneros y complejos de especies, sobre todo de regiones tropicales (véanse Jones, 1966; Riba y Butanda, 1987).

En 1982, Tryon y Tryon publicaron su obra Ferns and Allied Plants, en la que presentan una clasificación de las pteridofitas con especial referencia a las de América tropical, basada en la información publicada en las últimas décadas sobre el tema, y en la experiencia de los autores en el estudio de estas plantas. La obra contiene una sección acerca de la citología de los grupos, clave para familias de pteridofitas y, para cada una, descripción, comentarios acerca del grupo y clave para los géneros. Así mismo, para cada género, descripción, sistemática, ecología, distribución, palinología y citología, además de literatura pertinente al género. Esta es la obra taxonómica más extensa escrita hasta la fecha sobre pteridofitas tropicales.

Actualmente, las pteridofitas son estudiadas mediante estudios biosistemáticos (Gastony, 1991; Wagner, 1991; Werth, 1991). Dado que los sistemas de clasificación son en último término el resultado de la interpretación de la biología evolutiva de las pteridofitas, la aplicación del análisis electroforético en este campo es de considerable valor. La electroforesis proporciona información sobre la estructura del genoma, genera marcadores específicos para la detección de híbridos, se aplica al estudio de los procesos de cruzamiento y es muy útil en el análisis de los patrones de evolución (Haufler, 1985).

Un enfoque innovador es el de la aplicación de la cladística en relación a los fósiles, fundamentalmente Lycopsida (Bateman et al., 1992; Gensel, 1992).
Puede verse la aplicación de las computadoras en el campo de la taxonomía, en la elaboración de claves para la identificación de helechos en las Islas Británicas (HillCottingham y Moore, 1990) y Thelypteridaceae del sur de la India (Irudayaraj et al., 1993). En cuanto a la fase gametofítica, tenemos conocimiento sobre simulación de un modelo de crecimiento bidimensional (Montardy-Pausade, 1992).

Un análisis interesante lo constituye el desarrollo actual, en los últimos cinco años, de una escuela que se ha planteado el estudio de implicaciones filogenéticas mediante el análisis del DNA de cloroplastos. Esta escuela está encabezada por Soltis et al., (1991), Stein et al., (1991), Conant et al., (1992), Gastony et al., (1992), Horobe e Iwatsuki (1992) y Sandbrink et al., (1992) en diversos géneros como Polystichum, Pellaea, Adiantum, Platycerium y las Cyatheaceae.

\section{FLORÍSTICA}

De manera natural, los estudios resultantes de los avances taxonómicos han sido los florísticos, que han cubierto numerosas partes de la superficie terrestre como tratamientos regionales o como estudios de mayor cobertura. Sin mencionar a los del Viejo Mundo, en el continente americano se tiene información florística de Canadá y Estados Unidos (Lellinger, 1985), México (Knobloch y Correll, 1962; Smith en: Breedlove, 1981; Mickel y Beitel, 1988; Mickel, en: McVaugh, 1992; Pérez-García, 1993; Riba, 1993a, 1993b; Pacheco, 1994) (en las obras de Jones, 1966, y Riba y Butanda, 1979, está contenida una recopilación de publicaciones de tipo florístico de cobertura regional más restringida a la pteridoflora de México); Guatemala (Stolze, 1976, 1981, 1983); Costa Rica, Panamá y el Chocó (Lellinger, 1989), Perú (Tryon, 1964; Tryon y Stolze, 1989 y otros); Venezuela (Smith, lista de referencia, sin fecha); Ecuador (en proceso). La obra florística de mayor cobertura es la Flora Mesoamericana, que abarca desde el Sur de México hasta Panamá.

\section{ECOLOGÍA}

Hace algunas décadas, las publicaciones dedicadas a consideraciones sobre la ecología de pteridofitas eran muy escasas. Destaca por su importancia la contribución de Holttum (en: Verdoorn, ed., 1938), que básicamente está enfocada a las formas de vida. Actualmente la literatura es más vasta, y se encuentran estudios en los que se menciona a las pteridofitas en relación a condiciones de luz, humedad y temperatura en sus hábitats naturales. De estos trabajos los más relevantes son los de Parris (1985), Page (1979), Cousens et al., (1985), Farrar (1985), Gómez-P.(1985) y Kornás (1985).

Los trabajos de campo hechos por Farrar (1985), enfocaron aspectos relacionados con poblaciones de gametofitos perennes. Cousens et al., (1985) han considerado diferencias autoecológicas entre poblaciones de helechos de Estados Unidos; Peck (1980), Peck et al., (1990), Lindsay y 
Dyer (1990), Lindsay et al., (1992) y Werth y Cousens (1990), Pérez-García et al., (1982, 1994), han abordado estudios de banco de esporas y su significado en la biología de las pteridofitas. Finalmente, Riba y Reyes J. (1990) estudian la relación entre poblaciones de helechos y condiciones edáficas.

Estos estudios ayudan a comprender los diversos aspectos del ciclo de vida de las pteridofitas, así como sus adaptaciones de acuerdo con su capacidad de adecuarse al medio en que se establecen, y pueden estimular a los botánicos a profundizar en este campo, para entender las estrategias de las fases esporofítica y gametofítica de las pteridofitas de zonas tropicales y templadas y explicar su diversidad.

\section{CITOGENÉTICA}

Esta es una disciplina que surgió a fines de la primera mitad del siglo XX, y su principal pilar fue Manton (1950). Este investigador hizo a un lado las objeciones respecto al estudio citogenético de las pteridofitas que le plantearon otros investigadores, los que ponían como un obstáculo para interpretaciones adecuadas el tamaño y el número de sus cromosomas. Manton estableció técnicas y métodos que hasta la fecha son seguidos por numerosos investigadores. Los hallazgos de los citogenetistas han influido en la taxonomía al extremo de aplicar el concepto de «especie biológica» a poblaciones similares pero con diferente número cromosómico, aunque en nuestros días estas variantes citológicas son consideradas como variedades. Son bien conocidos los procesos apogámicos en los helechos, los cuales tienen alternancia de fases, pero ambas generaciones tienen el mismo número cromosómico como resultado de procesos compensatorios durante la meiosis. Mediante los estudios citogenéticos se sabe, por ejemplo, que la poliploidía es muy común, que las pteridofitas homospóricas tienen números cromosómicos básicos altos (20-50), mientras que los números básicos de cromosomas de las pteridofitas heterospóricas son bajos (8-11).

Entre los discípulos de Manton sobresalió Walker (1966), quién publicó un estudio citogenético sobre las pteridofitas de Jamaica, el más extenso de este tipo hasta la fecha, sobre las pteridofitas de los trópicos americanos. En este trabajo se analiza la citogenética de varios géneros y de un cierto número de complejos de especies e híbridos interespecíficos.

Las implicaciones taxonómicas de los estudios citogenéticos han ayudado a resolver problemas en este campo.

\section{ANATOMÍA}

Los estudios anatómicos de pteridofitas se han centrado básicamente en la organización del sistema vascular. En 1972, Ogura dio una descripción de la estructura anatómica de los órganos vegetativos de las pteridofitas. Destacan los trabajos de Beck (1970) y sus discípulos, de White (1963a, 1963b) y Hébant (1969), todos ellos influidos por la «Teoría del teloma» (Zimmerman, 1959). No debe dejarse a un lado la hipótesis de Bierhorst $(1968,1969)$ que sostiene que las pinnas de los helechos pueden haberse originado de enaciones como las que se encuentran en los tallos de Psilotum y Tmesipteris. Los patrones de venación foliar han sido importantes en la determinación de relaciones genéricas como lo demuestran las publicaciones de Pray $(1960,1962)$, Hara $(1962,1964)$ y Hagemann $(1964,1965,1967)$. Van Cotthem (1970) enfocó su investigación a los estomas en la hoja de los helechos estudiándolos en más de 200 géneros.

\section{PALINOLOGÍA}

La palinología de las pteridofitas ha sido poco estudiada de manera integral, comparativamente con la de las espermatofitas, aunque existen aportaciones eventuales de diversos autores. La primera obra de amplia cobertura en este campo es la de Erdtman y Sorsa (1971), quienes estudiaron algunos grupos representativos.

En su obra Spores of Pteridophyta, Tryon y Lugardon (1991) hicieron una descripción de las esporas de representantes de los diversos grupos de pteridofitas, de acuerdo con el uso de medios modernos de observación, como son el microscopio electrónico de transmisión (TEM) y el microscopio electrónico de barrido (SEM). Además de abordar los conceptos clásicos de forma, tamaño, estructura, superficie e inclusiones de las esporas, se presentan nuevos conceptos sobre el desarrollo y ultraestructura del esporodermo.

Por último, nos gustaría mencionar que como resultado de un simposio internacional sobre cultivo y propagación de pteridofitas que se verificó en 1992, Ide, Jermy y Paul (1993) editaron Fern Horticulture: Past, Present and Future Perspectives, en donde se tocan aspectos de propagación, sistemas de crecimiento artificial, micro-propagación, técnicas hortícolas, aspectos de conservación ex situ, banco de esporas y métodos de almacenamiento. Esto es interesante dado que los ecosistemas han sufrido un gran deterioro fundamentalmente por actividades antropogénicas, y el hecho de hablar de conservación de pteridofitas, nos enfrenta a pensar en las perspectivas en las que debemos enfocar nuestros esfuerzos para el estudio de este grupo de plantas. Este aspecto ha sido desarrollado por Pérez-García y Reyes J. (1993).

\section{CONSIDERACIONES FINALES}

Las referencias dadas en las páginas anteriores tienen la intención de presentar, de manera general y por sectores disciplinarios, las principales líneas abordadas en el estudio de las pteridofitas desde las primeras, que se basaban únicamente en los rasgos morfológicos de los esporofitos, con la intención de ubicarlas en los incipientes sistemas de clasificación, hasta las más modernas, en que ya se advierte una clara y necesaria interrelación disciplinaria. La revisión de la literatura citada muestra que la mayor parte de las publicaciones ha sido hecha por científicos europeos y estadounidenses, y la primera mención que se hace de un mexicano parece ser la de Rovirosa a principios de siglo (1909). No es sino hasta 1956 que Matuda, radicado en nuestro país desde 
1920, publica una relación florística de los helechos del Valle de México (1956a) y otra sobre los del estado de México (1956b). En la década de los sesenta, se inician las incursiones más frecuentes de científicos nacionales con publicaciones de diversa índole, principalmente orientadas a estudios de la pteridoflora nacional desde el punto de vista florístico. Es interesante mencionar que en las listas florísticas publicadas de manera consistente en diversas päìies de la República Mexicana, las pteridofitas no eran incluidas sino hasta fechas muy recientes, posiblemente debido a la falta de especialistas en este campo en México. Actualmente, la pteridoflora de nuestro país está siendo estudiada por botánicos nacionales en dos líneas principales: a) estudios florísticos a nivel nacional y a nivel regional con objeto de conocer el número y distribución de las especies en México (véase arriba, Florística) y, b) estudios morfogénicos de la fase sexual de helechos homospóricos, cuyos resultados permitirán fundamentar la homogeneidad de rasgos en especies de un género o en géneros afines (ver Pérez-García y Fraile 1986a, 1986b, Pérez-García y Reyes Jaramillo 1990, Reyes Jaramillo y Pérez-García 1991, 1994, Riba et al., 1992, Pérez-García y Riba 1993, Pérez-García et al., 1994.

Es claro que en México el estudio de las pteridofitas está restringido a estas dos líneas, y que faltan especialistas en la mayor parte de las disciplinas mencionadas anteriormente. Las perspectivas para el desarrollo de estos campos en nuestro país son pobres y su falta no será subsanada a corto plazo, a pesar de la necesidad de contar con especialistas que estudien desde distintos enfoques nuestra rica flora pteridofítica calculada entre 1,000 y 1,100 especies, distribuidas desde las regiones secas del noroeste hasta las ricas regiones tropicales húmedas del sur y sureste.

\section{LITERA TURA CITADA}

Adanson M. 1763. Familles des Plantes. 2 vols. Vincent. Paris. Agardh CA. 1822. Aphorismi Botanici. Parts 8 \& 9. Berlingianis. Lund Lit. Bateman RM, DiMichele WA, Willand, DA. 1992. Experimental cladistic analysis of anatomically preserved arborescent lycopsides from the Carboniferous of Euramerica: an essay on paleobotanical phylogenetics. Ann. Missouri Bot. Gard. 79: 500-559.

Beck CB. 1970. The appearance of the gymnosperms structure. Biol. Rev. 45: 379-400.

Berchtold FW, Presl JS.1820. O Prirozenosti Rostlin, aneb Rostlinar, Oddeleni 1. K.W. Anders. Prague.

Bierhorst DW. 1968. On the Stromatopteridaceae (fam. nov.) and on the Psilotaceae. Phytomorphology 18: 232-268.

Bierhorst DW.1969. On Stromatopteris and its ill-defined organs. Am. J. Bot. 56: 160-174.

Bower FO. 1908. The origin of a land flora. McMillan, London.

Bower FO. 1923-1928. The ferns (Filicales). 3 vols. University Press, Cambridge.

Brown R. 1810. Prodromus florae Novae Hollandiae et Insulae VanDiemen. Johnson, London.

Cesalpino A. 1583. De plantis libri, XVI. G. Marescottum. Firenze.

Conant DS, Stein DB, Valinski AE. 1992. Phylogenetic implications of chloroplast DNA restriction site variation in the Cyatheaceae. Am. J. Bot. 79: 117-118.

Cotthem W VAN. 1970. Comparative morphological study of the stomata in the Filicopsida. Bull. Jard. Bot. Natl. Belg. 40: 81-239. Cousens MI, Lacey DB, Kelly EM. 1985. Life history studies of ferns: a consideration of perspective. Proc. Roy. Soc. Edinburgh 86B: $371-380$.

Christensen C. 1905-1906. Index Filicum. Hagerup. Kobenhavn. i-lix.

Christensen C. 1913. Index Filicum, Supplementum (1906-1912). Hagerup. Kobenhavn.

Christensen C. 1917. Index Filicum. Supplement préliminaire pour les années 1913, 1914, 1915, 1916. Bogtrykkreri. Kobenhavn.

Christensen C. 1934. Index Filicum. Supplementum Tertium pro annis 1917-1933. Hagerup. Kobenhavn.

Dyer AF, edr. 1979. The experimental biology of ferns. Academic Press.

Dyer AF, Page CN, edrs. 1985. Biology of pteridophytes. Proc. Roy. Soc. Edinburgh 86. Edinburgh.

Dyer AF, Lindsay S. 1992. Soil spore banks of temperature ferns. Am. Fern J. 82: 89-122.

Erdtman G, Sorsa P. 1971. Pollen and spore morphology/plant taxonomy. Pteridophyta. Almkvist \& Wiksell/Stockholm.

Engler A, Prantl K. 1898-1902. Die natürlichen pflanzen-familien 1(4). Engelman. Leipzig.

Farrar DR. 1985. Independent fern gametophytes in the wild. Proc. Roy. Soc. Edinburgh 86B: 361-369.

Fée ALA. 1852. Genera Filicum (Cinquième Mémoire sur la famille des Fougères). Berger-Levrault. Strasbourg. Publicado anteriormente en 1850 en Mém. Soc. Mus. Hist. Nat. Strasbourg 4: 171-198.

Gastony GJ, Yatskievych G, Dixon CK. 1992. Chloroplast DNA restriction site variation in the fern genus Pellaea: phylogenetic relationships of the Pellaea glabella complex. Am. J. Bot. 79: 1072-1080.

Gensel PG. 1992. Phylogenetic relationships of the zosterophylls and lycopsida: evidence from morphology, paleoecology, and cladistic methods of inference. Ann. Missouri Bot. Gard. 79: 450-473.

Gómez-P LD. 1985. Ecology of some neotropical hybrid pteridophytes. Proc. Roy. Soc. Edinburgh 86B: 347-351.

Grew N. 1672. The anatomy of vegetables. Hickman. London.

Grew N. 1682. The anatomy of plants. Rawlins. London.

Hagemann W. 1964. Vergleichende Untersuchungen zur Entwicklungsgeschichte des Farnsprosses. I. Morphogenese und Histogenese an Sprosscheitel leptosporangiater Farnes. Beitr. Biol. Pflanzen. 40: 27-64.

Hagemann W. 1965. II. Die Blattentwicklung in der Gattung Adiantum L. Beitr. Biol. Pflanzen. 41: 405-468.

Hagemann W.1967. Bemerkungen zur Entwicklung und Differenzierung des Farnblatters und das Fiederwachstum von Regnellidium diphyllum Lind. Zeitschr. Pflanzenphysiol. 57: 26-45.

Hamilton RG. 1988. The significance of spore banks in natural populations of Athyrium pycnocarpon and Athyrium thelypterioides. Am. Fern J. 78: 96-104.

Hara N. 1962. Histogenesis of the venation in the pinnule of Mateuccia struthiopteris Todaro. Sci. Pap. Coll. Gen. Educ. Univ. Tokyo 12: $57-63$.

Hara N. 1964. Ontogeny of the reticulate venation in the pinna of Onoclea sensibilis. Bot. Mag. (Tokyo) 77: 381-387.

Haufler CH.1985. Pteridophyte evolutionary biology: the electrophoretic approach. Proc. Roy. Soc. Edinburgh 86B: 315-323.

Hébant C. 1969. Observations sur le phloème de quelques Filicinées tropicales. Naturallia Monspell. Sér. Bot. 20: 135-196.

Hill RH. 1971. Comparative habitat requirements of spore germination and prothallial growth of tree ferns in south eastern Michigan. Am. Fern J. 61: 171-182.

Hill-Cottingham P, Moore A. 1990. A computer key to the ferns of 
the British Isles. Pteridologist 2: 94 .

Holttum RE. 1938. The ecology of tropical pteridophytes. En: Verdoorn F, edr. Manual of pteridology. M. Nijhoff.The Hague.

Hooker WJ. 1838-1842. Genera Filicum. Bohn. London.

Horebe M, Iwatsuki K. 1992. Gene localization on the chloroplast DNA od the maiden-hair ferns: Adiantum capillus-veneris. Bot. Mag. (Tokyo) 105: 413-419.

Ide J M, Jermy AC, Paul AM. 1993. Fern horticulture, past, present and future perspectives. Intercept Ltd., Andover.

Irudayaraj V, Arockiadoss A, Manickam VS. 1993. Fernex: a computer experimental system for identifying south Indian Thelypteridaceae (ferns) members. Indian Fern J. 9: 274-281.

Jermy AC, Crabbe JA, Thomas BA, edrs. 1973. The Phylogeny and Classification of the Ferns. Bot. J. Linn. Soc. 67 (Suppl. 1): i-xiv.

Jones GN. 1966. An Annotated Bibliography of Mexican Ferns. University of Illinois Press. Urbana, Ill. xiii.

Kaulfuss GF. 1827. Das Wesen der Farrenkräuter besonders ihrer fruchttheile. Cnobloch. Leipzig.

Knobloch IW, Correll DS. 1962. Ferns and Fern allies of Chihuahua, Mexico. Texas Research Foundation. Renner, Texas.

Kornás J. 1985. Adaptative strategies of African pteridophytes to extreme environments. Proc. Roy. Soc. Edinburgh 86B: 391396.

Lazare JJ, Vivant J, Sastre C. 1991. Les ptéridophytes de Guadeloupe: biodiversité, éologie, protection. Bull. Soc. Bot. France, Actualités Botaniques 138: 197-214.

Lellinger DB. 1985. A Field Manual of the Ferns and Fern Allies of the United States and Canada. Smithsonian Institution Press. Washington, D.C.

Lellinger DB. 1989. The Ferns and Fern-allies of Costa Rica, Panama, and the Chocó (Part I: Psilotaceae through Dicksoniaceae). Pteridologia 2A.

Lindsay A, Dyer AF. 1990. Fern spore banks: implications for gametophyte establishment. En: Rita J, edr. Taxonomía, biogeografía y conservación de pteridófitos. Societat d'Historia Natural de les Iles Balears-IME, Palma de Mallorca, 243-253.

Lindsay S, Sheffield E, Dyer AF. Soil spore banks, fern conservation and isozyme analysis. The Fern Gazette (En prensa).

Linday S, Williams N, Dyer AF. Wet storage of fern spores: unconventional but far more effective? The Fern Gazette (En prensa).

Linnaeus C. 1753. Species Plantarum. 2 vols. Salvi. Stockholm.

Lira R, Riba R. 1993 (1994). Las pteridofitas (helechos y plantas afines) de México. Rev. Soc. Mex. Hist. Nat. (vol. esp.) 44: 99 -108 .

Malpighi, M. 1675. Anatomia Plantarum. Martyn. London.

Manton I. 1950. Problems of cytology and evolution in the Pteridophyta. Cambridge at the University Press.

Matuda E.1956a. Los helechos del Valle de México y alrededores. An. Inst. Biol. Mex. 27: 49-168.

Matuda E. 1956b. Los helechos del estado de México. Gobierno del estado de México. Dirección de Agricultura y Ganadería. Toluca, México.

Mettenius G. 1856. Filices Horti Botanici Lipsiensis. Voss. Leipzig.

Mettenius G. 1856. Ueber Einige Farngattungen. I. Polypodium. Abh. senckenb. naturforsch. Ges. 2: 1-138.

Mettenius G. 1858. Ueber einige farngattungen. II. Plagiogyria; III. Ueber die mitt einem Schleier versehenem Arten von Pteris; IV. Phegopteris und Aspidium. Abh. senckenb. naturforsch. Ges. 2: 265 - 420 .

Mettenius G. 1859. Ueber einige farngattungen. V. Cheilanthes; VI. Asplenium. Abh. senckenb. naturforsch. Ges. 3: 47-254.

Mickel JT. 1992. Pteridophytes. En: R. McVaugh R, edr. Flora Novogaliciana 17: 120-467.
Mickel JT, Beitel JM. 1988. Pteridophyte Flora of Oaxaca, Mexico. Mem. New York Gard. 46: 1 - 568.

Miller JH. 1968. Fern gametophytes as experimental material. The Bot. Rev. 34: 362 - 426.

Montardy-Pausade J. 1992. Computer simulation of bidimensional growth of the gametophyte of Anemia phyllitidis (L.) Swartz. Math. Biosci. 108: 219 -240.

Moore T. 1857-1862. Index Filicum. Pamplin. London.

Moran R, Riba R. edrs. Pteridofitas. En: Davidse G, Sousa M, Knapp S, edrs. Flora Mesoamericana. Vol.1. (En prensa).

Morison R. 1699. Plantarum Historiae Universalis Oxoniensis pars Tertia, post auctoris mortem expleta et absoluta a Jacobo Bobartio. Theatro Scheldoniano. Oxford.

Ogura Y. 1972. Comparative anatomy of vegetative organs of the Pteridophytes. Gebrüder Borntraeger. Berlin.

Pacheco L. 1994. Hymenophyllaceae. Flora de México 6: 1-56.

Page CN. 1979. Experimental aspects of fern ecology. En: Dyer AF, edr. The experimental biology of the ferns. Academic Press. London, 551-589.

Page CN, Dyer AF, Lindsay S, Mann DG. 1992. Conservation of pteridophytes: the ex-situ approach. En: Ide JM, Jermy AC, Paul AM, edrs. Fern horticulture, past, present and future. Perspectives Intercept Ltd., Andover.

Parris BS. 1985. Ecological aspects of distribution and speciation in Old World tropical ferns. Proc. Roy. Soc. Edinburgh 86B: 341346.

Peck, J.H. 1980. Life history and reproductive biology of the ferns of Woodman Hollow, Webster County, Iowa. Tesis Doctoral, Iowa State University, Ames, Iowa.

Peck JH, Peck CJ, Farrar DR. 1990. Influences of life history attributes on formation of local and distant populations. Amer. Fern J. 80: 126-142.

Pérez-García B. 1993. Marattiaceae. Flora de México 6: 1-12.

Pérez-García B, Fraile ME. 1986a. El gametofito de Holodictyum ghiesbrehgtii (Fourn.) Maxon. Biótica 10: 309-314.

Pérez-García B, Fraile ME. 1986b. El gametofito de Dicksonia sellowiana (Presl) Hooker. Biótica 11: 281-287.

Pérez-García B, Orozco-Segovia A, Riba R.1982. El banco de esporas de helechos en el suelo de Los Tuxtlas, Ver. Bol. Soc. Bot. México 43: 89-92.

Pérez-García B, Orozco-Segovia A, Riba R. 1994. The effects of white fluorescent light, far-red light, darkness, and moisture on spore germination of Lygodium heterodoxum (Schizaeaceae). Am. J. Bot. 81: 1367-1369.

Pérez-García B, Reyes Jaramillo I. 1990. Morfología del gametofito de Elaphoglossum petiolatum (Sw.) Urban (Lomariopsidaceae). Acta Bot. Mex. 10: 23-30.

Pérez-García B, Reyes Jaramillo I. 1993. Estrategias y perspectivas en la propagación y conservación de los helechos. Ciencias (Revista de difusión) 30: 11-19.

Pérez-García B, Riba R. 1993. Observaciones sobre los gametofitos de Woodwardia martinezii Maxon ex Weatherby y W. spinulosa Mart. \& Gal. (Blechnaceae). Acta Bot. Mex. 21: 7-14.

Pérez-García B, Riba R., Mendoza, A. 1994. Observaciones del gametofito de Thelypteris rhachiflexuosa Riba (Thelypteridaceae). Acta Bot. Mex. 28: 63-69.

Pérez-García B, Riba R. 1994 (1995). Bibliografia sobre gametofitos de helechos y plantas afines (1699-1991). Monogr. Syst. Miss. Bot. Gard. St. Louis, Missouri.

Pray TR. 1960. Ontogeny of the open dichotomous venation in the pinna of the fern Nephrolepis. Am. J. Bot. 47: 319-328.

Pray TR. 1962. Ontogeny of the closed dichotomous venation of Regnellidium. Am. J. Bot. 49: 464-472. 
Presl KB. 1825. Reliquiae Haenkeanae. I, part 1. Calve. Prague.

Presl KB. 1836. Tentamen Pteridographiae. Haase. Prague.

Radford AF, Dickison WC, Massey JR, Bell CR. 1974. Vascular plant systematics. Harper \& Row, Publishers.

Raghavan V. 1989. Developmental biology of fern gametophytes. Cambridge University Press.

Ratcliffe D.1990. Fearing for the future of the ferns. Weekend Telegraphy 14: 12 .

Ray J. 1686-1704. Historia plantarum. 3 vols. Clark. Loncion.

Reyes Jaramillo I, Pérez-García B. 1991. Desarrollo de los gametofitos de Thelypteris patens (Sw.) Small y de Thelypteris puberula (Baker) Morton var. puberula. Acta Bot. Mex. 16: 7-13.

Reyes Jaramillo I, Pérez-García B. 1994. Morfología y estrategias de reproducción del gametofito de Polypodium lepidotrichum (Fée) Maxon (Polypodiaceae). Acta Bot. Mex. 28: 71-78.

Riba R. 1993a. Lophosoriaceae. Flora de México 6: 13-16.

Riba R. 1993b. Metaxyaceae. Flora de México 6: 17-19.

Riba R, Butanda A. 1987. Bibliografía comentada sobre Pteridofitas de México. Consejo Nacional de la Flora de México, A.C.

Riba R, Pérez-García B,. Pérez-García M.1992. Schaffneria nigripes Fée (Aspleniaceae): morfogénesis del gametofito y anatomía y morfología del esporofito. Bol. Soc. Bot. México 52: 105-111.

Riba R, Reyes J I. 1990. Pityrogramma calomelanos (L.) Link in layers of volcanic ash in Los Tuxtlas, Veracruz, Mexico.Ann. Missouri Bot. Gard. 77: 287-289.

Rickard MH. 1991. Conservation - The fern story. En: Camus JM, edr. The history of British pteridology 1891-1991. Brit. Pterid. Soc. (sp. publ.) 4: 71-74.

Rovirosa JN. 1909. Pteridografía del sur de México, o sea, clasificación y descripción de los helechos de esta región, precedida de un bosquejo de la flora general. Imprenta de Ignacio Escalante. México.

Sandbrink JM, Van Han Roeland CHJ, Van Brederode J. 1992. Chloroplast DNA and morphological variation in the fern genus Platycerium (Polypodiaceae: Pteridophyta). Fern Gaz. 14: 97-118.

Smith AR. 1981. Pteridophytes. En: Breedlove DE, edr. Flora of Chiapas. Part 2. California Academy of Sciences.

Smith AR. Sin fecha. Pteridophytes of Venezuela, an annotated list. Department of Botany - Herbarium. University of California, Berkeley, California. Mimeógrafo.

Smith J. 1842-1843. An arrangement and definition of the genera of ferns, with observations on the affinities of each genus. J. Bot. 4: 38-56, 57-70, 147-168, 169-198.

Soltis PS, Soltis DE, Wolf DG. 1991. Allozymic and chloroplast DNA analysis of polyploid in Polystichum (Dryopteridaceae). I. The origins of $P$. californicum and $P$. scopulinum. Syst. Bot. 16: 245-256.
Stein DB, Conant DS, Valinski AE. 1991. Phylogenetic implications of chloroplast DNA variation in the Cyatheaceae. Am. J. Bot. 78 (supl. 6): 154.

Stokey AG.1918. Apogamy in the Cyatheaceae. Bot. Gaz. (Crawfordsville) 65: 97-102.

Stolze RG. 1976. Ferns and Fern Allies of Guatemala. Part I. Ophioglossaceae through Cyatheaceae. Fieldiana, Botany 39: 1-130.

Stolze RG.1981. Ferns and Fern Allies of Guatemala. Part II. Polypodiaceae. Fieldiana, Botany n.s. 6: 1-522.

Stolze RG.1983. Ferns and Fern Allies of Guatemala. Part III. Marsileaceae, Salviniaceae and the fern allies (including a comprehensive index to parts I, II and III). Fieldiana, Botany n.s. 12: 1-91.

Strasburger E.1894. Ueber periodische Reduction der Chromosomenzahl im Entwicklungsgang der Organismen. Biol. Zbl. 14: 817-852.

Tryon AF, Lugardon B. 1991. Spores of the Pteridophyta. SpringerVerlag.

Tryon R. 1964. The ferns of Peru. Polypodiaceae (Dennstaedtieae to Oleandreae). Contr. Gray Herb. Harv. Univ. 144: 1-253.

Tryon RM, Stolze RG. 1989. Pteridophyta of Peru. Part I. 1. Ophioglossaceae - 12. Cyatheaceae. Fieldiana, Botanyn.s. 20: 1-145.

Tryon RM, Stolze RG. 1989. Pteridophyta of Peru. Part II. 13. Pteridaceae - 15. Dennstaedtiaceae. Fieldiana, Botany, n.s. 22: $1-128$.

Tryon RM, Stolze RG. 1991. Pteridophyta of Peru. Part IV. 17. Dryopteridaceae. Fieldiana, Botany n.s. 27: 1-176.

Tryon RM, Stolze RG. 1992. PTeridophyta of Peru. Part III. 16. Thelypteridaceae. Fieldiana, Botany n.s. 29: 1-80.

Tryon RM, Stolze RG. 1993. Pteridophyta of Peru. Part V. 18. Aspleniaceae - 21. Polypodiaceae. Fieldiana, Botany n.s. 32: 1-190.

Tryon RM, Tryon AF. 1982. Ferns and allied plants, with special reference to Tropical America. Springer-Verlag, New York.

Walker TG. 1966. A cytotaxonomic survey of the pteridophytes of Jamaica. Trans. Roy. Soc. Edinburgh 66: 179-237.

Werth CR, Cousens MI. 1990. Summary: the contributions of population studies on ferns. Am. Fern J. 80: 183-190.

White RA. 1963a. Tracheary elements of the ferns. I. Factors which influence tracheid length; correlation of length with evolutionary divergence. Am. J. Bot. 50: 447-455.

White RA. 1963b. Tracheary elements of the ferns. II. Morphology of tracheary elements; conclusions. Am. J. Bot. 50: 514-522.

White RA. 1971. Experimental studies of the sporophyte of ferns. BioScience 21: 271-275.

Zimmerman W. 1959. Die phylogenie der Pflanzen. Gustav Fischer, Stuttgart. 